\title{
Visible Light Emitting Waveguide on Si Chip
}

\author{
Iago R. Diez ${ }^{\mathrm{a}, \mathrm{b}}$, I.J. Luxmoore ${ }^{\mathrm{b}}$, G.R. Nash ${ }^{\mathrm{b}}$, and A. Baldycheva ${ }^{\mathrm{b}}$ \\ aEngineering and Physical Sciences Research Council Centre for Doctoral Training in \\ Metamateriales, University of Exeter, Exeter, EX4 4QF, United Kingdom \\ ${ }^{\mathrm{b}}$ College of Engineering, Mathematics and Physical Sciences, University of Exeter, Exeter, \\ EX4 4QL, United Kingdom
}

\begin{abstract}
Photonic lab-on-a-chip portable platforms have proved to be very sensitive, rapid in analysis and easy-to-use. However, they still rely on a bulk light source to operate, thus hindering the actual portability and potential for commercial realization. In the present paper we have proposed a design for a light emitting structure that could be easily implemented on chip. The design consists of a $\mathrm{Si}_{3} \mathrm{~N}_{4}$ strip waveguide on $\mathrm{SiO}_{2}$ substrate, with an active material that emits light as top and lateral cladding. The cross-section of the waveguide was optimised to support both excitation and emission as guided modes, with a high mutual overlap and high confinement to the cladding. This ensures an efficient light emission activation from the cladding and a stable propagation along the waveguide. The proposed structure shows to be operative along the visible range; demonstrated from $400 \mathrm{~nm}$ to $633 \mathrm{~nm}$. The procedure we have followed along this report can be virtually used for designing the cross-section geometry of any strip waveguide system so that the performance is optimised for a given cladding refractive index and emission and excitation wavelengths. In addition we have proposed the use of polymeric quantum dots as the gain material to be used as active cladding. The ease of on-chip integration of this gain material via spin-coating, together with the simplicity of our light emitting waveguide, makes our light source design suitable for large-scale integration on Si chip. Specially, for lab-on-chip applications where multiplexed operation is essential.
\end{abstract}

Keywords: Silicon Photonics, Light source, Light emitting waveguide, Lab on a chip, Active cladding, Polymeric quantum dots

\section{INTRODUCTION}

Lab-on-a-chip (LOC) are miniaturised platforms that integrate one or several laboratory functions on the same chip. Example of these are: sample preparation, analysis, detection, data processing and signal delivery. ${ }^{1}$ This technology is very promising for the field of bio-chemical and medical analysis. One of the reasons is that the enhanced reactivity of chemicals in the microscale results in an increase of sensitivity, a faster response time and diagnosis, and enables real-time process control and monitoring, while using low volume samples.

The ultimate goal of LOC technology is to achieve a fully-portable platform that can provide an efficient, rapid diagnosis outside of the laboratory. Therefore, there exists a need of integrating on chip all the devices and components that are conventionally necessary to perform the whole analysis in the laboratory.

In particular, the combination of LOC platforms with integrated photonics is very promising due to their high capacity of miniaturization, robustness, reliability and potential multiplexing at low cost and potential for mass production. In addition, integrated photonics offers a solution for combining chemical, optical, microfluidics and electronics functions in one single LOC platform. ${ }^{2}$ This technology allows to replace bulky, costly and complex equipment such as interferometers ${ }^{3,4}$ spectrometers $^{5}$ and biosensors ${ }^{2}$ for their equivalent micro-engineered and integrated system that are compact, low cost, and easy to use.

The vital component of any chip relying on integrated photonics is the laser source which is used for the operation of the chip. Therefore, the integration of the laser source on chip is of extreme importance for the whole industry. From among the numerous advantages that an on-chip laser provide, we may remark the larger

Further author information: Send correspondence to Iago R.Diez by email to ir274@exeter.ac.uk 
energetic efficiency in terms of output power while keeping a very low footprint. In addition, the coupling of light from an on-chip light source to adjacent waveguides is more efficient than the conventional cumbersome coupling methods relying on optical fibres out of the chip.

In spite of its huge relevance, there still exists a lack of an easy and simple integration method on chip that is compatible with complementary metal oxide semiconductor (CMOS) technology for large-scale manufacturing, while keeping a good performance. Therefore, the big majority of LOC still rely on external light sources. This technological issue is even more pronounced for the visible wavelength range because the features of the used structures need to be typically below 100nm-size, which is within the range of resolution limit of standard large-scale fabrication equipment (mainly based on optical lithography).

The implementation of a laser source, or in general a light source, on chip is inherently dependent on the integration method that its gain medium requires. In the next paragraphs we will discuss the typical active materials used for light sources nowadays.

A direct implementation coming from the microelectronics industry are the epitaxially-grown self-assembled semiconductor quantum dots. These materials emit light by the radiative recombination of the electron-hole pair (exciton). An example of this is the wurtzite $I n_{x} G a_{1-x} N$ quantum dots in a GaN matrix. ${ }^{6}$ However, these type of quantum dots require an epitaxial growth via atomic layer deposition or molecular beam epitaxy. Both fabrication processes involve a machinery that is not affordable for every laboratory. Also, the lattice constant mismatches between adjacent layers and difference in thermal expansion coefficients result in high density of defects that lowers the performance of these sources. ${ }^{7}$

A gain medium easier to implement with LOC technology are the organic dyes. Fluorescent organic materials provide a very broad range of molecules with different luminescent spectra, therefore being very versatile. Also, another advantage of organic compounds is the easy integration on chip which can be done by spin-coating or physical vapour deposition. However, their major drawback is the photodegradation (photobleaching) after a short time which lowers the lifetime of the device. ${ }^{8}$

A hybrid approach that combines organic and semiconductor technologies are the colloidal or polymeric quantum dots. These are inorganic nanocrystals, whose size ranges from $\sim 2 \mathrm{~nm}$ to $10 \mathrm{~nm}$, that are normally dispersed in a polymeric matrix. The semiconductor chemical nature of the quantum dots provides a bandgap that produces an emission by the recombination of the electron-hole pair. They posses outstanding properties: a broad absorption spectra with large absorption cross-section, but sharp emission (typically Full Width Half Maximum 30-40nm). ${ }^{8}$ In addition, the emission due to band gap can be tuned by varying the size of the nanocrystal. These last features makes them suitable for a multiplexed system: a single excitation wavelength could excite different-sized quantum dots. ${ }^{9}$ Also, when compared to organic dyes, quantum dots show great photostability, reduced photobleaching and very high quantum yields. ${ }^{8}$ Due to all these reasons, the quantum dots seem to be the promising choice for achieving an easy-to-integrate and efficient light source on chip.

In this paper, we propose a design for an on-chip light emitting structure that was devised with the aim of achieving: 1) A simple structure whose gain material can be easily integrated via a back-end CMOS compatible process, and 2) with small footprint, but energy-efficient enough for LOC purposes. The design is described in the following section.

\section{DESIGN AND METHODS}

\subsection{System Description: Light Emitting Waveguide}

The proposed light emitting structure consists of a strip waveguide made of a thin $\mathrm{Si}_{3} \mathrm{~N}_{4}$ core on $\mathrm{SiO}_{2}$ substrate, with a gain material as top and lateral claddings. This active cladding is ultimately covered by $\mathrm{SiO}_{2}$. This gain material is suitable for light emission when optically excited. The schematic of the design can be seen in Fig. 1.

The working principle of this system is as follows: the waveguide is designed to support propagating modes of light that are not strongly confined to the waveguide core so that a large fraction of the mode leaks to the active cladding. If along this waveguide we send a mode of a wavelength (pump) capable of exciting the molecules of the active cladding, this gain medium will emit light (signal) that would be coupled to the correspondent 

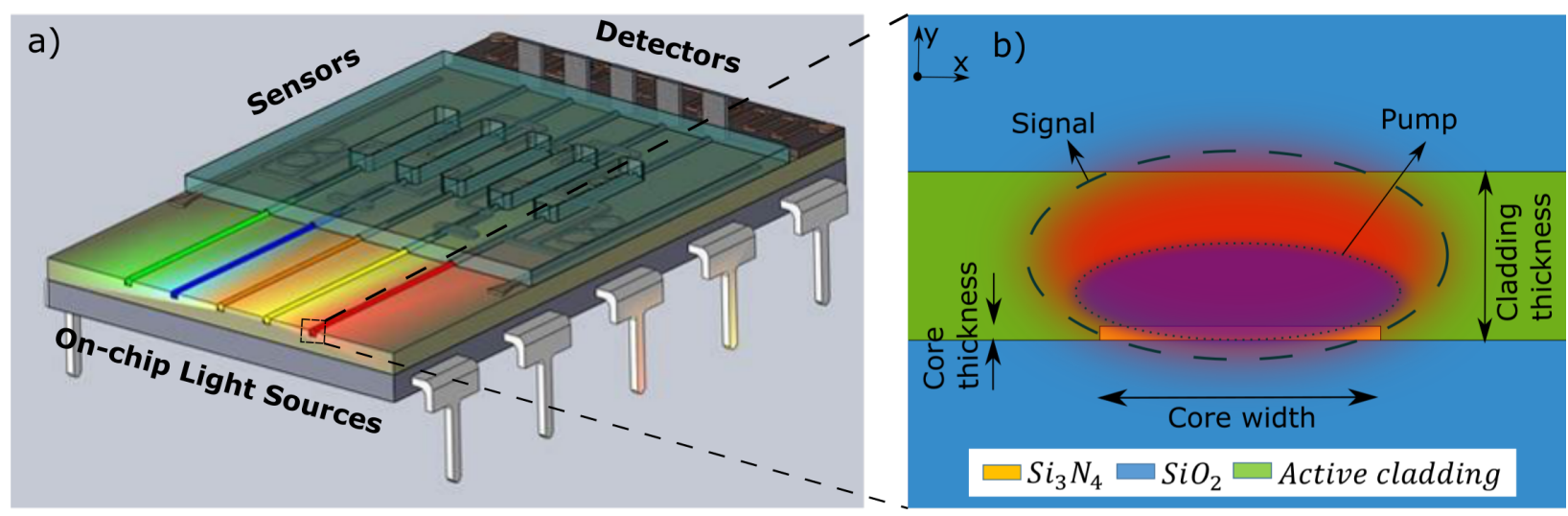

Figure 1. a) Schematic of a photonic lab-on-a-chip with multiple integrated light emitting waveguides. b) Schematic of the strip waveguide cross-section with a representation of the pump and signal guided modes. The characteristic geometrical parameters that were optimised are indicated in the figure.

propagating mode at this emission wavelength. By "mode", we refer to a stable distribution or "shape" of the electric and magnetic field that propagates along the waveguide.

We chose $\mathrm{Si}_{3} \mathrm{~N}_{4}$ for the passive core of the waveguide because this material ensures robust designs on Si-chip, shows high transparency along the whole visible range (i.e. low losses) and, when compared to polymers or $\mathrm{SiO}_{2}$, a higher refractive index . These characteristics makes $S i_{3} N_{4}$ suitable for guiding the light along the chip. ${ }^{10-13}$

The selection of gain material for the proposed active cladding was the polymeric quantum dots due to their ease of integration on chip and outstanding luminescent properties. However, the only characterising parameter of the gain material implemented in the simulation is the refractive index. Therefore, the design was optimised for any gain material of the same refractive index considered (discussed in Subsection 2.3).

Other light emitting waveguides based on polymeric quantum dots were also reported by other authors. ${ }^{14,15}$ The main difference with respect to our design is that in their structure the polymeric active region acts as the core (air-cladded), instead of the cladding.

\subsection{Characterization Parameters}

The system will be characterised by three parameters: the cladding confinement factor, the pump-signal overlap and the effective mode area. The efficiency of this light emitting structure can be estimated from the first two parameters. ${ }^{11,16}$

The power confinement factor (from now on, just confinement factor) accounts for the fraction of the guided mode power that propagates through the active cladding region along the waveguide, and is defined as: ${ }^{17}$

$$
\gamma_{\text {clad }}=\frac{\iint_{\text {gain }}\left|<S_{z}(x, y)>\right| d x d y}{\iint_{\text {all }}\left|<S_{z}(x, y)>\right| d x d y}
$$

where $\left\langle S_{z}(x, y)>\right.$ is the time-averaged z-component of the Poynting vector. The numerator integration is done only in the region of the active cladding (denoted as "gain") whereas the denominator integration is done all over space (denoted as "all"); both integrals restricted to the cross section, i.e. (x,y)-plane (See Fig. 1). Values may range between 0 and 1 .

The overlap factor between the guided modes at the pump and signal wavelengths accounts for the common spatial region between their respective distributions of the electric field, and is defined as ${ }^{11,18}$

$$
\eta_{p-s}=\frac{\left(\int_{\text {gain }}\left|\overrightarrow{E_{p}^{*}}(x, y) \overrightarrow{E_{s}}(x, y)\right| d x d y\right)^{2}}{\int_{\text {gain }}\left|\overrightarrow{E_{p}}(x, y)\right|^{2} d x d y \int_{\text {gain }}\left|\overrightarrow{E_{s}}(x, y)\right|^{2} d x d y}
$$


where $\overrightarrow{E_{p}}(x, y)$ and $\overrightarrow{E_{s}}(x, y)$ are the pump and signal in-plane electric field, respectively, evaluated in the (x,y) plane. Values may range between 0 and 1.

The performance of our system in terms of the activation of light emission can be estimated by the product of the confinement factor for the emission mode we want to excite (i.e. signal) and the overlap between this emission mode and the excitation mode (i.e. pump). Therefore, our figure of merit (FOM) will be:

$$
F O M=\eta_{p-s} \gamma_{c l a d, s i g n a l}
$$

The effective mode area characterises the spatial extent of each mode in the gain medium, and therefore it estimates the area of the output signal at the end of the waveguide. This parameter can be defined as ${ }^{19}$ :

$$
A_{e f f}=\frac{\left(\iint_{\text {all }}\left|<S_{z}(x, y)>\right| d x d y\right)^{2}}{\iint_{\text {gain }}\left(\left|<S_{z}(x, y)>\right|\right)^{2} d x d y}
$$

\subsection{Simulation Methods}

The system was simulated by a finite elements method software (COMSOL Multiphysics, commercially available). We performed an eigenfrequency study using the "Mode analysis" tool to obtain the electric field and power distributions of the guided modes throughout the cross-section of the waveguide. The simulation domain is enclosed by walls where Perfect Electric Conductor boundary conditions are applied.

A guided mode of light, i.e. an electric field profile that can propagate along the waveguide, is entirely determined by the waveguide geometry and refractive index values of the materials. That is why, the waveguide core thickness and width, and cladding thickness were designed to maximise the interaction of pump and signal with the active cladding $\left(\gamma_{c l a d}\right)$ as well as the pump-signal overlap $\left(\eta_{p-s}\right)$ while maintaining the mode guided along the whole structure. The refractive index of the active cladding was fixed to 1.7, which is a possible value for a polymer. Since the refractive index of polymeric quantum dots is the one for the host polymer, this implies that the study here presented can be virtually applied to any wavelength within the visible range emitted by the quantum dots with the correspondent size.

This strip waveguide design was optimized considering the following operation wavelengths: $\lambda_{p}=400 \mathrm{~nm}$ for the excitation wavelength (pump) and $\lambda_{s}=513 \mathrm{~nm}$ for the emission wavelength (signal). These values were selected because they are at the edge $\left(\lambda_{p}\right)$ and at the middle $\left(\lambda_{s}\right)$ of visible-light range, respectively. However at the end of the paper, we also show the electric field distribution for the modes at the wavelengths of the typical laser sources lines, as a proof of the practicability of our design within the visible range. These wavelengths are: $488 \mathrm{~nm}\left(\mathrm{Ar}^{+}\right.$-ion), $532 \mathrm{~nm}$ (Nd:YAG), 633nm (He-Ne).

We may remark some simulation considerations before showing the obtained results:

- For a trustworthy calculation of the modes distribution and parameters, the walls where the boundary conditions of Perfect Electric Conductor are applied need to be far from the waveguide so that the mode is not distorted.

- Only the Transversal Electric (TE) and Transversal Magnetic (TM) fundamental modes were calculated*.

- We have considered the wavelength dependence of the refractive index for the $\mathrm{SiO}_{2}$ substrate and cover, and for the $\mathrm{Si}_{3} \mathrm{~N}_{4}$ waveguide core ${ }^{\dagger}$. Just to give a rough idea of the values we used: $n_{\mathrm{Si}_{3} \mathrm{~N}_{4}} \approx 2.03$ and $n_{\mathrm{SiO}_{2}} \approx 1.48$ over the visible range. No absorption was taken into account.

- Regarding the graphs in this paper, it may be important to say that only the marker-points are the values extracted from the simulations. The lines joining the markers are artificially introduced to give a clearer vision of the tendencies.

\footnotetext{
${ }^{*}$ We may remind that there are not pure TE or TM modes in dielectric waveguides. Therefore the modes are "quasiTE" or "quasi-TM". Just for the sake of simplicity we will still call them TE or TM.

${ }^{\dagger}$ Data from refractiveindex.info
} 


\section{RESULTS AND DISCUSSION}

The procedure taken for the optimization is shown in the order of execution.

\subsection{Core Thickness}

The waveguide $S i_{3} N_{4}$ core thickness (or simply waveguide thickness) was varied from $30 \mathrm{~nm}$ to $500 \mathrm{~nm}$. In this first simulation the $\mathrm{SiO}_{2}$ cover was not considered. The width of the waveguide was chosen to be $1000 \mathrm{~nm}$. As substrate and cladding are very large compared to the core of the waveguide, the mode will be confined when the effective refractive index of the mode is larger than the bulk values of cladding and substrate $n_{e f f}>$ $\max \left(n_{\text {clad }}, n_{\mathrm{SiO}_{2}}\right)=1.7$.

Results are shown in Fig. 2, only for those values that gave a solution for a guided mode. The solution for $500 \mathrm{~nm}$ is not shown because the mode was too confined to the core of the waveguide yielding a $n_{\text {eff }} \approx 2$, i.e. roughly the bulk value for $S i_{3} N_{4}$.

We see that the confinement to the cladding $\gamma_{c l a d}$, for both TE and TM modes, is larger for thinner cores, consequently the $n_{\text {eff }}$ is smaller and approaches the bulk value $n_{\text {clad }}$. The TE modes are more bound to the waveguide core since they require a thinner waveguide to show the same $\gamma_{c l a d}$ as the TM modes.

We have selected the edge value of $50 \mathrm{~nm}$ of waveguide thickness since this value guarantees the maximum $\gamma_{\text {clad }}$ for TE while maintaining the mode guided. This is only true for TE modes. However, also TM modes can exist for this small core thickness but upon adding the cover over the cladding.

\subsection{Cladding Thickness}

Now we will include the $\mathrm{SiO}_{2}$ cover over the active cladding to confine more the modes in the gain medium. The cladding thickness was varied from $100 \mathrm{~nm}$ to $1000 \mathrm{~nm}$; results are shown in Fig. 3.

From claddings thicker than 300 $\mathrm{nm}$, both signal and pump (for TE and TM) modes are mainly confined in the active cladding $\left(\gamma_{\text {clad }}>0.5=50 \%\right)$. This confinement increases with the thickness until a certain value from which $\gamma_{\text {clad }}$ is practically constant, i.e. the mode cannot spread further into the cladding while being guided by the waveguide region. The $n_{\text {eff }}$ of each mode shows the same behaviour, rising up to values closer to the bulk $n_{\text {clad }}$, implying that the modes are increasingly becoming more "cladding modes", i.e. not so bound to the $\mathrm{Si}_{3} \mathrm{~N}_{4}$ core.
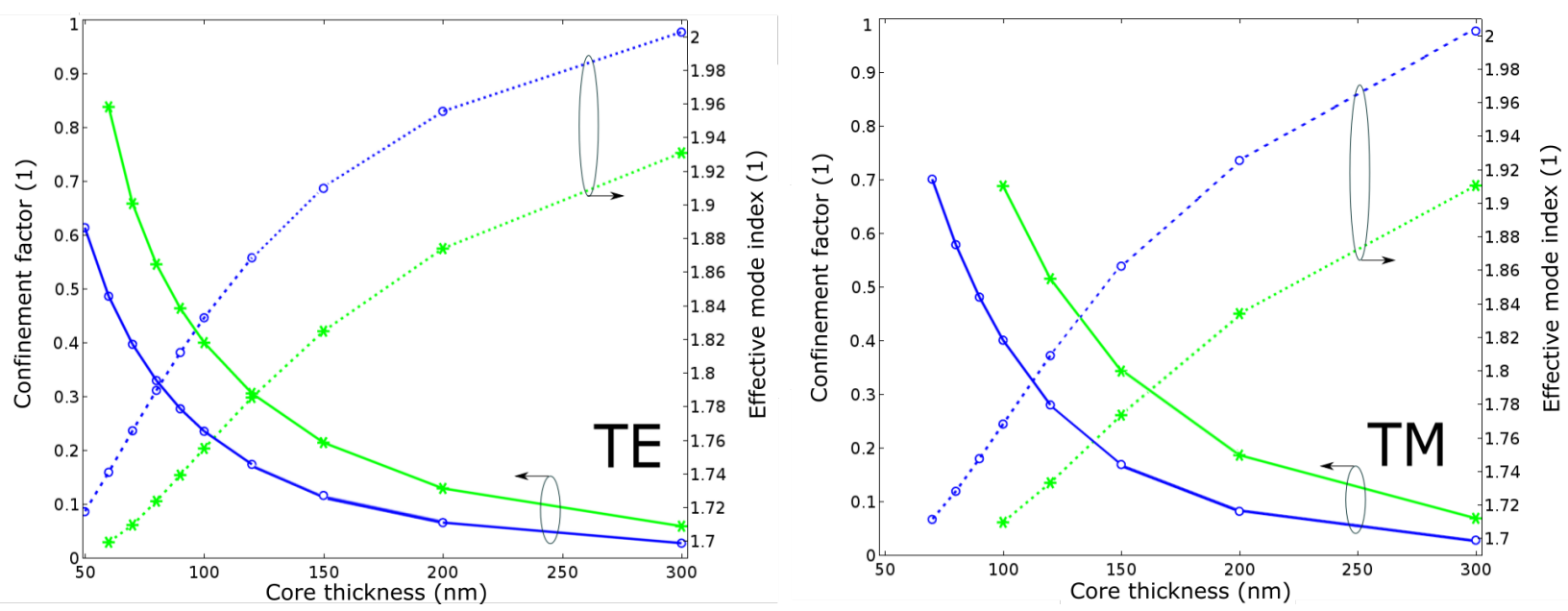

Figure 2. The variation of the effective refractive index $n_{\text {eff }}$ and confinement factor $\gamma_{c l a d}$ of signal $\lambda_{s}=513 \mathrm{~nm}(*)$ and pump $\lambda_{p}=400 \mathrm{~nm}$ (o) with respect to the waveguide core thickness; for TE (left image) and TM (right image) modes. 

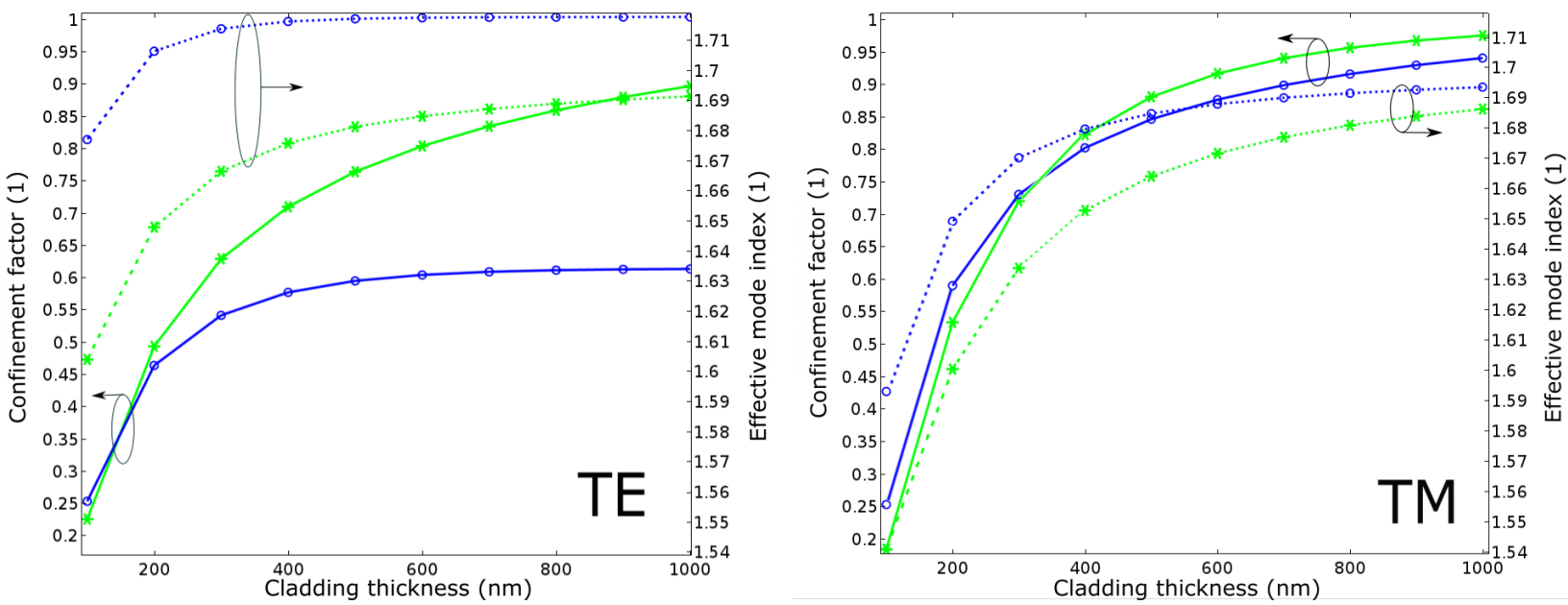

Figure 3. The variation of the effective refractive index $n_{\text {eff }}$ and confinement factor $\gamma_{c l a d}$ of signal $\lambda_{s}=513 \mathrm{~nm}(*)$ and pump $\lambda_{p}=400 \mathrm{~nm}$ (o) with respect to the cladding thickness; for TE (left image) and TM (right image) modes.
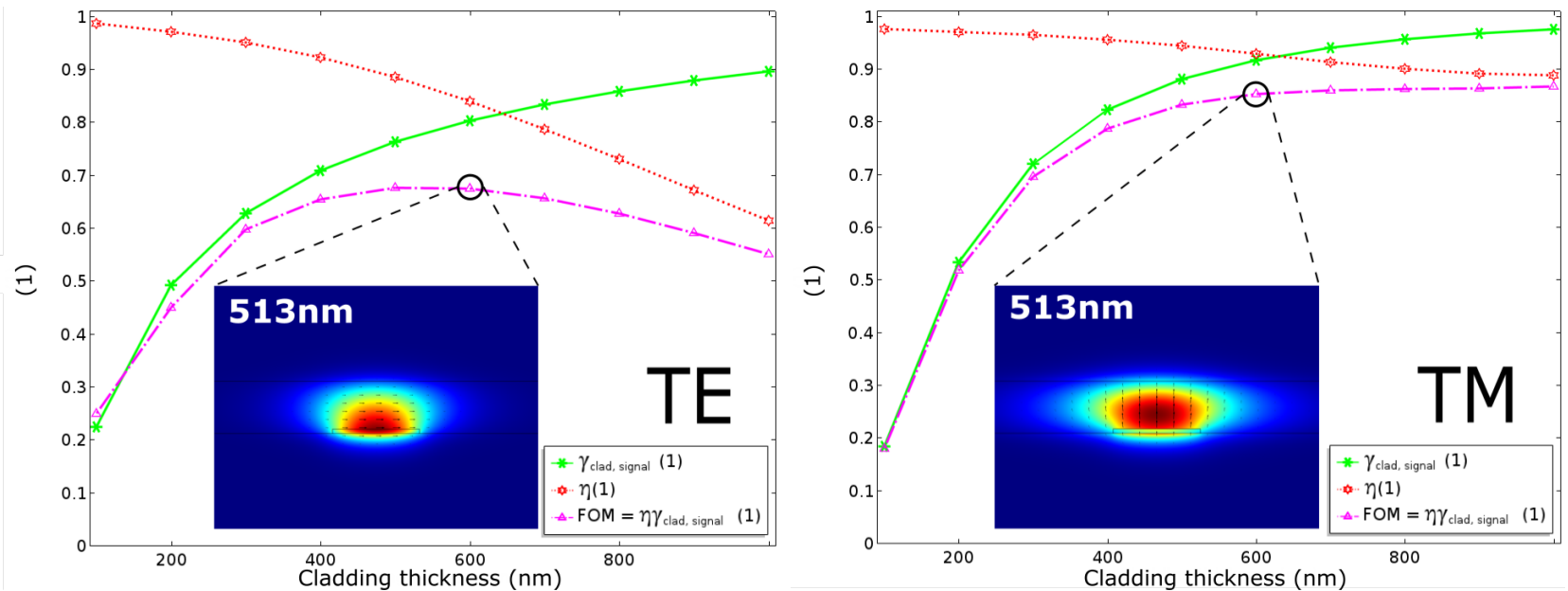

Figure 4. The variation of the confinement factor $\gamma_{\text {clad }}$ of signal $(*)$, the pump-signal overlap $\eta(\star)$, and figure of merit FOM $(\triangle)$ with respect to the cladding thickness; for TE (left image) and TM (right image) modes. The inset in both graphs represents the modulus (colour map) and direction (arrow field) of the in-plane electric field distribution of the signal mode $\left(\lambda_{s}=513 n m\right)$. The colour scale goes from minimum value in dark blue up to maximum value in dark red.

For determining the cladding thickness that maximises the system performance, the pump-signal overlap $(\eta)$ and figure of merit (FOM) were calculated; results are shown in Fig. 4. The overlap decreases for larger cladding thickness; this decay is more pronounced for TE modes. It is worth mentioning that the difference of confinement between pump and signal (see Fig. 3) seems to be correlated with their overlap value.

The $F O M$ for TE presents a maximum $F O M_{T E} \approx 0.676=67.6 \%$ at $500 \mathrm{~nm}-600 \mathrm{~nm}$ thickness, whereas for TM the value remains nearly constant $F O M_{T M} \approx 0.853=85.3 \%$ from a thickness value of $600 \mathrm{~nm}$. Due to this different behaviour, we have selected our optimised thickness value to be $600 \mathrm{~nm}$ since it maximises $F O M_{T E}$ without lowering $F O M_{T M}$. From another point of view, taking into account that our system can work independently with TE and TM modes we can estimate the overall performance to be $F O M_{t o t}=\frac{1}{2}\left(F O M_{T E}+\right.$ $\left.F O M_{T M}\right)$. This value is maximised for a cladding thickness of $600 \mathrm{~nm}$. 


\subsection{Waveguide Width}

The final geometrical parameter varied was the width of the waveguide core from $200 \mathrm{~nm}$ to $2000 \mathrm{~nm}$. The signal (pump) confinement for the TE mode decay from a value $\approx 92 \%(81 \%)$ at $300 \mathrm{~nm}$ to $\approx 78 \%(60 \%)$ from $2000 \mathrm{~nm}$ onwards. However, the pump-signal overlap increases at the same rate the signal confinement decays so that the FOM remains practically constant for the whole range considered. Regarding the TM mode, there is barely any change in the pump and signal confinement values, but it is worth mentioning that a minimum core width of $800 \mathrm{~nm}$ is required for the existence of the signal mode. Therefore, we decided to keep the value of $1000 \mathrm{~nm}$ width for making sure this TM mode exists.

\subsection{Optimized Design}

The geometrical parameters of the optimised cross-section of the strip waveguide are summarised in Table 1. The characterization parameters for the resulting TE and TM mode operating with a pump wavelength at $400 \mathrm{~nm}$ and a signal wavelength at $513 \mathrm{~nm}$, are summarised in Table 2. In this table we have added the values for the effective mode area of the pump and signal modes to estimate the area of the output light that would be emitted from the edge of the waveguide. In our opinion, the small sizes obtained, $0.753 \mu m^{2}$ for TE and $0.890 \mu m^{2}$ for TM, would make the output light of the waveguide suitable as a light spot for LOC analysis.

Table 1. Selected parameters for the strip waveguide system operating with an excitation at 400nm and emission at 513nm.

\begin{tabular}{|llll|}
\hline Waveguide thickness & Waveguide width & Cladding thickness & Cladding refractive index \\
\hline $50 \mathrm{~nm}$ & $1000 \mathrm{~nm}$ & $600 \mathrm{~nm}$ & 1.7 \\
\hline
\end{tabular}

Table 2. Characterization parameters of the operating TE and TM modes of the strip waveguide system; obtained for an excitation at $400 \mathrm{~nm}$ (pump) and emission at 513nm (signal).

\begin{tabular}{|l|llll|}
\hline Mode & $\gamma_{\text {clad }, s}$ & $\eta_{p-s}$ & $F O M$ & $A_{\text {eff }, s}$ \\
\hline TE & $80.5 \%$ & $84 \%$ & $67.6 \%$ & $0.753 \mu m^{2}$ \\
TM & $91.8 \%$ & $93 \%$ & $85.3 \%$ & $0.890 \mu m^{2}$ \\
\hline
\end{tabular}

\subsection{Visible range operation}

As a demonstration of the viability of the proposed design for the whole visible spectra, we have represented the TE modes at the lines of the typical commercially available laser sources: $488 \mathrm{~nm}, 532 \mathrm{~nm}, 633 \mathrm{~nm}$. See Fig. 5. From the images, it can be intuited that the closer the wavelength pump and signal are, the higher the overlap between them; therefore larger efficiency.

Here we only show the TE modes for these wavelengths. However, the TM modes show the same behaviour. In fact, for TM modes the overlap values are much higher than for TE modes. It is worth mentioning that, a minimum core thickness of $60 \mathrm{~nm}$ is necessary so that the TM mode at $633 \mathrm{~nm}$ is guided. 


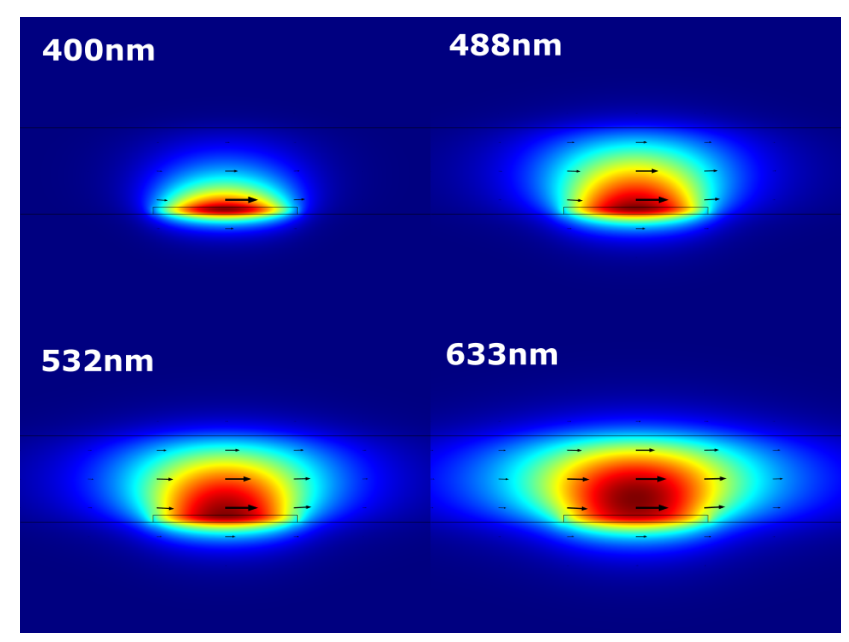

Figure 5. The modulus (colour map) and direction (arrow field) of the in-plane electric field distribution (TE mode) for the following wavelengths: $400 \mathrm{~nm}, 488 \mathrm{~nm}, 532 \mathrm{~nm}$ and $633 \mathrm{~nm}$. The colour scale goes from minimum value in dark blue up to maximum value in dark red.

\section{CONCLUSION}

In the present paper we have proposed a design for a light emitting waveguide. This structure consists of a strip waveguide made of $\mathrm{Si}_{3} \mathrm{~N}_{4}$ core on $\mathrm{SiO}_{2}$ substrate, with an active material as top and lateral cladding. The structure is finally covered by $\mathrm{SiO}_{2}$ layer. This active cladding is the gain medium that emits the light (signal) upon absorbing the adequate excitation wavelength (pump). The waveguide was designed to support both the pump and signal as guided modes, with a high mutual overlap and high confinement to the cladding.

We have shown that our specific selection of geometrical parameters makes the system operative along the visible range (demonstrated from $400 \mathrm{~nm}$ to $633 \mathrm{~nm}$ ), for the refractive indices of the before-mentioned materials and active cladding (here assumed to be 1.7). Since the refractive index of polymeric quantum dots is the one of the polymer matrix, and this value could be 1.7; automatically our design could work for any visible wavelength once the proper quantum dots are selected.

The procedure we have followed along this report can be virtually used for designing the cross-section parameters of any strip waveguide that optimises its performance, for a given active cladding (refractive index) and signal and pump wavelengths. Once selected the wavelength for the signal, the performance of the system increases for pump wavelengths spectrally closer to the signal wavelength. This is only constrained by the specific absorption and emission spectra of the active cladding.

The target application for this combination of gain material and simple waveguide structure was lab-on-a-chip platforms where multiplexed systems are very useful for parallel operation. In addition, the ease of integration of the polymeric quantum dots via spin-coating makes the design suitable for large-scale manufacturing and at low cost.

\section{ACKNOWLEDGMENTS}

This research was possible thanks to the Engineering and Physical Sciences Research Council (EPSRC) Centre for Doctoral Training in Electromagnetic Metamateriales at University of Exeter (Grant No. EP/L015331/1) and also via the EPSRC Grant EP/N035569/1.

\section{REFERENCES}

[1] J.V.Jokerst, J., D.Bhagwandin, Floriano, P., N.Christodoulides, and McDevitt, J. Anal. Chem. 82(5), 1571 (2010). 
[2] M.C. Estevez, M. A. and Lechuga, L., "Integrated optical devices for lab-on-a-chip biosensing applications," Laser Photonics 6(4), 463-487 (2012).

[3] B. Sepulveda, J. S., Ro, D., Moreno, M., Blanco, F., Mayora, K., Domnguez, C., and Lechuga, L., "Optical biosensor microsystems based on the integration of highly sensitive machzehnder interferometer devices," J. Opt. A: Pure Appl. Opt. 8, S561-S566 (2006).

[4] K. Qin, S. H., Retterer, S., Kravchenko, I., and Weiss, S., "Slow light machzehnder interferometer as label-free biosensor with scalable sensitivity," Opt. Lett. 41(4) (2016).

[5] S.J. Sweeney, Y. Z. and Goodyer, I., "The development of a novel monolithic spectrometer chip concept," in [Integrated Optics: Devices, Materials, and Technologies XVI], J. E.Broquin, G., ed., Proc. SPIE 8264 (2012).

[6] S. Tomic, J. P., Migliorato, M., Young, R., and Vukmirovic, N., "Visible spectrum quantum light sources based on $\mathrm{In}(\mathrm{x}) \mathrm{Ga}(1-\mathrm{x}) \mathrm{N} / \mathrm{GaN}$ quantum dots.," ACS Photonics 2, 958963 (2015).

[7] Zhou, Z., Yin, B., and Michel, J., "On-chip light sources for silicon photonics," Light Sci. Appl. 4, - (2015).

[8] Alivisatos, A., Gu, W., and Larabell, C., "Quantum dots as cellular probes," Annu Rev Biomed Eng. 7, 55-76 (2005).

[9] Smith, A. and Nie., S., "Semiconductor nano- crystals: Structure, properties, and band gap engineering," Acc. Chem. Res. 43(2), 190-200 (2010).

[10] Baets, R., Subramanian, A., Clemmen, S., Kuyken, B., Bienstman, P., Le Thomas, N., Roelkens, G., Thourhout, D., Philippe, H., and Severi, S., "Silicon Photonics: silicon nitride versus silicon-on-insulator.." in Optical Fiber Communication Conference, OSA Technical Digest (online) (Optical Society of America, 2016),. Paper Th3J.1.

[11] Purnawirman, Li, N., Magden, E., Singh, G., Singh, N., Baldycheva, A., Hosseini, E., Sun, J., Moresco, M., Adam, T., Leake, G., Coolbaugh, D., Bradley, J. B., and Watts, M., "Ultra-narrow-linewidth Al2O3 : Er3+ lasers with a wavelength-insensitive waveguide design on a wafer-scale silicon nitride platform," Opt. EXPRESS 25(12), 13705-13713 (2017).

[12] Li, N., Purnawirman, P., Magden, E., Singh, G., Singh, N., Baldycheva, A., Hosseini, E., Sun, J., Moresco, M., Adam, T., Leake, G., Coolbaugh, D., Bradley, J., and Watts, M., "Ultra-narrow-linewidth erbium-doped lasers on a silicon photonics platform," in [Silicon Photonics XIII], Reed, G. and Knights, A., eds., Proc. SPIE 10537, 10537121-7 (22 February 2018).

[13] Faneca, J., Hogan, B., Alonso, E., Craciun, M., and Baldycheva, A., "2D materials integrated in Si3N4 photonics platform," in [Silicon Photonics XIII], Reed, G. and Knights, A., eds., Proc. SPIE 10537, 1053701-12 (22 February 2018).

[14] Gordillo, H., Suarez, I., Abargues, R., Rodriguez-Canto, P., Albert, S., and Martinez-Pastor, J. P., "Polymer/qds nanocomposites for waveguiding applications," Journal of Nanomaterials 2012, 960201 (2012).

[15] Shen, K., S. Baig, G. J., Paik, Y., Kim, S. J., and Wang, M., "Broadband infrared light emitting waveguides based on uv curable pbs quantum dot composites," in [Optical Interconnects XVIII], J. E.Broquin, G., ed., Proc. SPIE 10538, 105380L (22 February 2018).

[16] Hosseini, E., Purnawirman, Bradley, J. B., Sun, J., Leake, G., Adamand, T., Coolbaugh, D., , and Watts, M., "CMOS-compatible $75 \mathrm{~mW}$ erbium-doped distributed feedback laser," Opt. Lett. 39(11), 3106-3109 (2014).

[17] Passaro, V. and Notte, M., "Optimizing SOI slot waveguide fabrication tolerances and strip-slot coupling for very efficient optical sensing," Sensors 12, 24362455 (2012).

[18] Paschotta, R., [Encyclopedia of Laser Physics and Technology: Article on mode-matching], Wiley-VCH.

[19] Afshar, S. and Monro, T., "A full vectorial model for pulse propagation in emerging waveguides with subwavelength structures," Opt. EXPRESS 17(4), 2298-2318 (2009). 